\title{
Expressões faciais de emoção em bebês: importância e evidências
}

\section{Babies' facial expressions of emotion: importance and evidences}

\section{Deise Maria Leal Fernandes Mendes*}

Doutoranda do Programa de Pós-Graduação em Psicologia Social da Universidade do Estado do Rio de Janeiro - UERJ, Rio de Janeiro, RJ, Brasil

\section{Maria Lucia Seidl de Moura**}

Professora titular de Psicologia Social, Instituto de Psicologia da Universidade do Estado do Rio de Janeiro - UERJ, Rio de Janeiro, RJ, Brasil

\begin{abstract}
Resumo
As expressões emocionais de bebês têm despertado crescente interesse, refletindo a grande importância atribuída a suas habilidades para lidar com emoções. Sua capacidade de produzir e reconhecer expressões faciais de emoção parece central para se compreender o desenvolvimento infantil, seja cognitivo, afetivo ou social. Com o objetivo de favorecer futuros trabalhos, discutem-se as lacunas encontradas na literatura voltada para expressões faciais de emoção em bebês, e a conveniência de se adotar uma perspectiva sociocultural e evolucionista na formulação de hipóteses e produção de novos estudos empíricos. Fica evidente a necessidade de mais investigações realizadas em ambiente natural e de estudos longitudinais.
\end{abstract}

Palavras-Chave: Expressões emocionais, Expressões faciais, Afetividade, Sorriso, Interação mãe-bebê.

\begin{abstract}
The emotional expressions of babies have provoked increasing interest, reflecting the importance attributed to their abilities to deal with emotions. Babies' capacity to produce and to recognize facial expressions of emotion seems central to understand infant cognitive, affective or social development. Aiming to presenting some orientations to new studies in this area, identified gaps in the literature are discussed, and the adoption of a socio-cultural and evolutionary perspective for new investigations is proposed. From those discussions it is evident the necessity of more studies focusing the first year of life, produced in natural context at home and with longitudinal designs.
\end{abstract}

Keywords: Emotional expressions, Facial expressions, Affectivity, Smile, Motherinfant interaction. 
A capacidade dos bebês de produzirem e reconhecerem expressões faciais de emoção tem sido considerada como elemento central para a compreensão do desenvolvimento infantil, nos seus aspectos cognitivo, afetivo e social. As teorias contemporâneas sobre desenvolvimento doself, afeto e cognição sublinham a importância das interações interpessoais e têm se interessado muito pelo seu aspecto afetivo e emocional (WALKERANDREWS, 1997). Buscam ampliar o conhecimento científico sobre as emoções nos bebês, tanto no que diz respeito à produção e percepção de expressões emocionais. Os processos e habilidades envolvidos têm sido vistos como essenciais para o estabelecimento e a qualidade das interações iniciais que, por seu turno, constituem-se em contexto propício ao desenvolvimento emocional e da intersubjetividade (ROCHAT, 2001; TRONICK, 1989).

Com este trabalho pretende-se discutir as lacunas encontradas na literatura voltada para expressões faciais de emoção em bebês, e a conveniência de se adotar uma perspectiva sociocultural e evolucionista na formulação de hipóteses e produção de novos estudos empíricos. Para tanto, de início, será apresentado um panorama das publicações na área com foco no primeiro ano de vida, período tido como abrangendo os principais marcos do desenvolvimento emocional na primeira infância. A partir de uma análise deste quadro, objetiva-se apontar carências de informação e sugerir linhas de atuação para futuras pesquisas.

De fato, as emoções talvez constituam o fenômeno psicológico mais evidente para os seres humanos. Contudo, seu estudo e de suas expressões, na psicologia, passou por períodos de altos e baixos, tendo apresentado, inclusive, momentos de quase completo abandono. Nas últimas três décadas, entretanto, assiste-se a uma crescente retomada do tema como objeto de formulações teóricas e pesquisas empíricas. O interesse pela sua ontogênese também pode ser medido pelo aumento do número de estudos que abordam a expressão emocional em bebês e crianças maiores.

Buscas a bases de dados eletrônicas revelam quantitativos que podem ser tomados como referencial. De acordo com o Web of Science, por exemplo, somente entre 2000 e 2006, foram publicados sete vezes mais estudos sobre emoção (9.383), do que em todos os anos das décadas de 1970 e 1980 juntos (1.352), e dois terços a mais do que se produziu entre 1990 e 1999 (5.577). Uma pesquisa recente ao Google Scholar, permitiu a recuperação de mais de 50.000 .000 de documentos de caráter diverso, sobre o assunto.

Quando o foco é um tema mais específico nesse campo, como o sorriso em bebês, a tendência a uma curva ascendente no interesse dos estudiosos, também é clara. Assim, no Psyclnfo, em sua seção PsycArticles, são referidos 150 artigos produzidos entre 1989 e 1999, e 94 somente entre 2000 e 2006. No Eric (Education Resources 
Information Center), todos os artigos encontrados foram publicados a partir de 1978. Finalmente, do Web of Science, constam 19 estudos realizados entre 2000 e 2006, 18 entre 1990 e 1999, e apenas 2 no período de 1970 a 1989.

O interesse crescente pelas expressões emocionais de bebês parece ser reflexo da importância atribuída às suas habilidades para expressar e lidar com emoções e afetos. Essas manifestações, considerando-se as limitações do repertório de comportamentos de um bebê, ocorrem, especialmente, através das expressões faciais. Nesse sentido, é importante lembrar que as primeiras exposições a expressões emocionais de outras pessoas ocorrem, em geral, no contexto familiar, no qual os bebês participam de trocas afetivas e observam respostas dos pais a eles e a outros parceiros.

Os pais, como principais cuidadores, assumem o papel de responsáveis pela estruturação do ambiente (físico e social) e definição de rotinas diárias para seus filhos. Essas práticas são coerentes com as expectativas que têm em relação a eles, e o estilo de criação e de socialização que desejam adotar (HARKNESS; SUPPER, 1992). Desse modo, transmitem às crianças formas de ver o mundo em que vivem e de se relacionar com outras pessoas. Parece, portanto, que é no microsistema familiar (BRONFENBRENNER, 1996) que as crianças começam a aprender a lidar com as emoções.

Partindo dessas considerações preliminares, assume-se o pressuposto de que estudar a ontogênese das expressões emocionais é essencial à compreensão dos mecanismos de produção, regulação e percepção de emoções e do desenvolvimento emocional dos seres humanos. Acredita-se ainda, que o ambiente familiar e, mais especificamente, as interações iniciais mãe-bebê sejam o contexto privilegiado para a manifestação e aprimoramento das habilidades no campo afetivo e de interação social. Através das trocas afetivas da mãe com o bebê, ele vivencia calor emocional, o começo de um processo de conhecimento do outro e a possibilidade de expressão de suas emoções. Regulações mútuas entre os parceiros criam padrões dinâmicos e organizados de comportamentos e respostas afetivas (SEIDL DE MOURA; RIBAS; SEABRA et al. no prelo).

Importantes avanços têm sido feitos, recentemente, no estudo das emoções em bebês e da natureza da comunicação emocional entre bebês e adultos. As emoções e comunicações emocionais dos bebês são muito mais organizadas do que previamente se pensava (Cohn; Tronick, 1987; Kaye ; Fogel, 1980). Eles apresentam uma variedade de expressões afetivas discretas que é apropriada para a natureza de eventos e seu contexto (IZARD, FANTAUZZO, CASTLE et al., 1995). Também apreciam o significado emocional da aparência afetiva e demonstrações de afetividade de seus cuidadores (WEINBERG; TRONICK, 1994). 
Ao longo das últimas três décadas, numerosos trabalhos têm sido realizados com a preocupação de entender melhor o desenvolvimento emocional de bebês. Sem dúvida, estabelecem um patamar de conhecimento e indicam direções para novos estudos. Caracterizam-se, freqüentemente, por ocorrerem em ambiente de laboratório e se valerem de situações estruturadas de comunicação face-a-face. Muitos deles têm se dedicado a investigar as reações emocionais de bebês em situações diversas em que interagem com um parceiro. Em geral, trata-se de interações em que participam a mãe e/ou o pai e, a depender dos objetivos, uma pessoa estranha. Também são freqüentes os estudos que se valem do paradigma da face imóvel, que será descrito adiante.

Um traço marcante é a predominância de pesquisas voltadas para as expressões faciais de emoções positivas, que pode ser atribuída à natureza dos objetivos e do próprio objeto de estudo. Como argumenta Messinger (2002), a investigação de expressões emocionais negativas envolve sérios entraves. Respeitados os princípios da ética em pesquisa, expressões de emoções negativas discretas, como raiva e desconforto, são difíceis de serem eliciadas em bebês. Outro aspecto também mencionado é o de que o sorriso é facilmente reconhecido por juízes ingênuos como uma expressão de alegria, nas etapas iniciais do desenvolvimento e posteriormente. Já a face de choro, associada a emoções negativas, incorpora características tanto da expressão de raiva quanto de desconforto, o que dificulta a discussão de questões essenciais acerca da relação entre emoção e expressão facial.

Alguns temas são recorrentes, como a reação do bebê a comportamentos contingentes (BIGELOW, 1998; BIGELOW, MACLEAN; MACDONALD, 1996), sua sensibilidade para demonstrações afetivas do parceiro (MONTAGNE; WALKER-ANDREWS, 2002), o papel da sensibilidade dos pais na expressão afetiva dos bebês (BRAUNGART-RIEKER, GARWOOD et al., 1998; BRAUNGART-RIEKER; GARWOOD; POWERS et al, 2001) e a influência do ambiente familiar no seu desenvolvimento emocional, sobretudo em casos com diagnóstico de quadro de depressão em algum dos cuidadores (CAMPBELL; COHN ; MEYERS, 1995; COHN; CAMPBELL; MATIAS et al, 1990). Além destes, há os que investigam o papel de um componente afetivo e emocional na manifestação e desenvolvimento dessa habilidade (KAYE; FOGEL, 1980; LEGERSTEE; VARGHESE, 2001; STACK; MUIR, 1990). Nesse âmbito, destacam-se os que cuidam estritamente da percepção e produção de expressões faciais de emoções, sendo privilegiado o sorriso como expressão de afeto positivo (DICKSON; WALKER; FOGEL, 1997; MESSINGER; FOGEL ; DICKSON, 2001).

Uma seleção desse acervo, em função de interesses e orientação teórica, permite que se tenha um panorama da produção científica específica, se reflita a respeito dos resultados alcançados e se projetem possíveis desdobramentos e novos estudos. As principais evidências que trazem em 
termos das conquistas do bebê, ao longo de seu primeiro ano de vida, podem ser organizadas cronologicamente, fornecendo um quadro sintético do seu desenvolvimento emocional nessa etapa inicial. Antes, porém, parece oportuno mencionar algumas opções metodológicas e procedimentos ou paradigmas que se tornaram marcantes em pesquisas com essa natureza.

\section{Paradigmas e procedimentos}

Dentre os paradigmas e procedimentos comumente utilizados destaca-se o da "face imóvel" (FI). Nesse procedimento, o adulto, geralmente a mãe, recebe a instrução de, após algum tempo de interação com o bebê, congelar sua face e parar de falar com o bebê. Diante dessa situação, observam-se as reações do bebê. Deste modo, é possível estudar o efeito de alterações das expressões emocionais do adulto no comportamento da criança que com ele interage. Os estudos que se valem deste paradigma mostram a importância da dinâmica das expressões faciais do adulto durante as interações face-a-face. No entanto, como lembram D'Entremont e Muir (1997), o período em que o adulto permanece com a face imóvel também envolve perda ou interrupção da contingência, um importante fator nas interações bebê-adulto. Deste modo não se pode isolar totalmente o efeito da ausência de expressões.

Tronick (1989) sugere que durante interações, o bebê aprende a se comunicar com um adulto através da regulação conjunta de sinais emocionais. Ao longo da interação, adultos e bebês exprimem sinais um para o outro na forma de mensagens afetivas. Cada um altera seu comportamento em resposta a esses sinais para alcançar os objetivos de uma interação positiva. Quando o adulto se apresenta com a face imóvel, frustra a tentativa do bebê de interagir, causando stress.

Um outro procedimento comum a uma parcela dos estudos examinados cria uma situação estruturada de interação face-a-face, em que mães e bebês interagem através de monitores de tv. O bebê é colocado sentado, diante de um monitor de tv. Uma câmera filma o bebê. A mãe também senta diante de um outro monitor que projeta a imagem de seu bebê naquele momento, isto é, a imagem do bebê é direcionada para o monitor da mãe. Uma segunda câmera filma a mãe olhando para seu bebê na tela e direciona a sua imagem para o monitor do bebê.

A configuração desse ambiente permite às mães olharem seus bebês durante a interação, e aos bebês verem a vídeo-imagem da mãe, de modo a dar a ambos a impressão de estarem mantendo um contato visual. Comumente, os filmes são apresentados ao bebê em duas condições, ao vivo e em replay, com o objetivo de verificar se estabelecem distinção entre elas e se reagem diferentemente a cada uma. 
Os estudos sobre percepção de expressões faciais, segundo Alonso, Molina, Serrano e Carriba (2004), têm utilizado, com freqüência, dois outros procedimentos em que a medida selecionada tem sido o tempo de fixação do olhar. Estes procedimentos são os paradigmas de habituação visual e de preferência visual. O primeiro deles se inicia com uma fase de familiarização em que uma expressão facial é exibida um número de vezes suficiente para que o tempo de fixação visual decresça. A esta, sucede uma outra fase, de discriminação, na qual se apresenta uma expressão facial distinta. Espera-se que no caso do bebê perceber a diferença entre a expressão a que se habituou e a nova, o tempo de fixação visual a esta última aumente.

No procedimento de preferência visual, duas imagens de uma mesma expressão facial são exibidas simultaneamente, por várias vezes. Quando decresce a fixação às duas expressões, uma delas é mudada e se verifica se há um incremento no tempo em que o bebê olha para a expressão nova. Com base no paradigma de habituação, assume-se que o aumento no tempo de fixação significa que o bebê estabelece alguma diferença entre as duas expressões faciais, demonstrando preferência pela nova imagem.

No entanto, a discriminação dos traços expressivos característicos das emoções não constitui um indicador direto da habilidade para reconhecer o significado afetivo que compreendem as expressões faciais. Assim, outros estudos têm investigado essa questão através de procedimentos específicos que requerem a percepção categorial da expressão. Cada expressão facial deve ser percebida como uma categoria diferenciada, muito mais por seus traços distintivos que possuam valor para comunicar o afeto, do que por outras características dos estímulos (D'ENTREMONT; MUIR, 1997; HAVILAND; LELWICA, 1987).

Um outro método ressalta aspectos da percepção de emoções nos bebês que não costumavam ser investigados de forma sistemática. Trata-se da capacidade de uma percepção intermodal, a partir da associação entre imagem e som (voz humana). São utilizados como estímulo filmes sonoros com expressão facial de determinadas emoções (alegria e raiva, por exemplo). Os filmes são exibidos de forma a serem acompanhados por uma única expressão vocal (o som de fundo) que corresponda afetivamente a uma das expressões faciais tratadas. Investiga-se a percepção da informação afetiva, como ela ocorre e se é mantida ou se varia, em função do olhar dos bebês. Se estabelecerem uma associação entre som e imagem, tenderão a olhar mais fixamente para a expressão facial correspondente ao som apresentado. Variações de situações e condições são introduzidas em alguns estudos, como por exemplo, alterações na informação referente à sincronia e velocidade (por edição ou apresentando a voz com defasagem de alguns segundos da imagem). 
Quanto à capacidade de bebês de expressarem emoções, segundo Alonso e cols. (2004), a maior parte dos estudos tem utilizado dois tipos de procedimentos: julgamentos realizados por adultos e códigos de observação da conduta facial. Os estudos de julgamento são os que têm maior tradição e consistem na avaliação do estado emocional apresentado pelo bebê, a partir de sua expressão facial. Os estudos que empregam códigos de observação, também chamados estudos de componentes expressivos, permitem que um observador especializado possa codificar as mudanças faciais que caracterizam uma determinada expressão emocional. Entre os códigos de observação geralmente usados, destacamse o FACS (Facial Action Coding System) de Ekman e Friesen, o MAX (Maximally Discriminative Facial Movement Coding System) de Izard ou a versão do FACS para bebês, denominada BabyFACS de Oster (ALONSO; cols., 2004; IZARD; cols., 1995).

\section{Expressões faciais de emoção: estudos empíricos}

A partir da revisão da literatura foi possível verificar que os estudos sobre expressão emocional em recém-nascidos são escassos. Do conjunto selecionado, além do realizado por Kawakami, Takai-Kawakami, Tomonaga, Suzuki, Kusaka; Okai (2007), apenas o de Messinger e cols. (2002) destaca-se também nesse aspecto. Os demais, em sua grande maioria, contemplam idades a partir dos três meses, aproximadamente. Tal característica, aliada ao reduzido número de estudos longitudinais, deixa uma lacuna. Há carência de evidências empíricas relativas ao período que vai do nascimento aos dois a três meses. Mais crítico ainda, há carência de resultados que, ao contemplarem um intervalo de tempo e não idades específicas, contribuam mais diretamente para inferências em termos da ontogênese do desenvolvimento socioemocional.

Uma síntese das principais evidências relatadas nessas investigações é apresentada a seguir. Estas evidências estão organizadas de forma a se tentar esboçar um cenário das expressões de afeto positivo no decorrer do primeiro ano de vida, seguindo uma cronologia das idades dos bebês. Elas constituem uma base para algumas reflexões que, espera-se, sirvam como orientação para novos trabalhos.

Evidências de uma estabilidade morfológica de expressões emocionais básicas em bebês, desde as etapas iniciais, falam a favor de uma condição inata. Contudo, a maneira de se expressar, freqüências e durações, sentimentos associados e significados parecem passar por mudanças ao longo do desenvolvimento e variar de acordo com o contexto e o parceiro. Assim, condicionantes sociais e culturais criam particularidades, e acredita-se que influenciem o desenvolvimento emocional. 
A se considerar as manifestações emocionais positivas, há relatos da presença de expressões de sorriso nos bebês humanos, desde os primeiros momentos de vida. De acordo com os resultados obtidos por Messinger e cols. (2002) com bebês de 55 horas de nascidos em média (de 5 a 106 horas de vida), os recém-nascidos não apenas sorriem, como uma parcela considerável deles ( $50 \%$ dos bebês estudados) apresenta um tipo de sorriso comum entre crianças mais velhas e adultos, o sorriso Duchenne bilateral. Alguns inclusive (em $1 / 8$ dos bebês estudados), com nível de intensidade considerado maduro. Um aspecto importante a se salientar é que, nestes casos, os bebês foram filmados enquanto dormiam. Não se tem indicação de estudo com registro de sorriso em recém-nascidos, com algumas horas ou poucos dias de vida, em momentos em que estivessem acordados.

Essas manifestações de sorriso em estado de sono ou sonolência, também chamadas de sorrisos endógenos ou espontâneos, ocorrerem sem a presença de qualquer estímulo externo identificável. Entretanto, é a partir de dois meses, aproximadamente, que algumas transformações profundas parecem ocorrer com relação à exibição dessa expressão emocional e às habilidades sociais dos bebês. Segundo Rochat (2001) e Rochat e Striano (1999), é um período marcado pela emergência de um novo senso de self como agente no ambiente e do senso de experiência compartilhada (intersubjetividade primária). Caracteriza-se ainda, de acordo com os autores, pela reciprocidade com o outro, como parte de uma nova perspectiva, contemplativa, adotada pelo bebê em relação às pessoas. Para esses estudiosos, esta é a primeira transição chave no desenvolvimento sociocognitivo inicial. Ao assumir esta perspectiva, o bebê começa a perceber que o outro é como eu porque responde com otiming apropriado e demonstrações emocionais.

Aos dois meses, os sorrisos do bebê e de sua mãe começam a se mostrar como comportamentos organizados de maneira contingente. Nessa fase, observa-se, comumente, que as mães se valem de expressões faciais para tentar atrair a atenção dos filhos e eliciar expressões positivas. Entre seis e 18 semanas, cresce a freqüência de expressões positivas dirigidas à mãe, seja a partir de sua tentativa de eliciar essas respostas, seja como iniciativa dos bebês (COHN ; TRONICK, 1987). Antes disso, de acordo com Kaye e Fogel (1980), esses comportamentos ainda não se mostram contingentes, já que as expressões faciais da mãe raramente eliciam respostas positivas dos bebês. Porém, sem elas, essas respostas não eram observadas, o que pode indicar um processo em curso.

A emergência de novos padrões de interação é documentada por Lavelli e Fogel (2005). Os autores relatam mudanças no comportamento interativo observado nos bebês, que variou de um ciclo entre olhar dirigido a um ponto qualquer e atenção simples para a face da mãe, nas primeiras semanas, a uma seqüência complexa de transições durante o segundo 
mês. Estas transições se constituíam de episódios de atenção concentrada, sorriso e expressão facial associada à emissão de sons (como arrulhos) dispostos em seqüências de comunicação positiva.

Em torno dos dois a quatro meses, embora o tempo em que os bebês olham para a mãe decresça, nesses episódios de interação face-a-face, eles mostram um aumento de expressões de afeto positivo (VAN BEEK; HOPKINS; JOEKOMA, 1994). Passam a reagir cada vez mais, com sorrisos responsivos e contingentes aos sorrisos de sua mãe (BIGELOW, 1998; BIGELOW; COLS., 1996; SYMONS; MORAN, 1994). Há indicações de que suas habilidades sociais em situações de comunicação ampliam-se em função dos comportamentos maternos. Dos participantes da investigação de Legerstee e Varghese (2001), aqueles com mães que demonstraram mais afeto, exibiram níveis mais elevados de comportamentos pró-sociais e expectativas sociais. Independentemente da direção de seu olhar, bebês se engajam mais em sorrisos quando suas mães estão sorrindo do que quando não estão (KAYE; FOGEL, 1980).

Os bebês aos dois meses parecem conseguir discriminar mudanças nas características faciais que denotam expressão emocional (NELSON; HOROWITZ, 1983), distinguindo expressões de alegria, tristeza e raiva e exibindo expressões compatíveis para alegria e raiva. Suas respostas são aparentemente adequadas às emoções expressas pela mãe (Haviland; Lelwica, 1987), que demonstram capacidade em evocar reações específicas em seus bebês (LANDAU, 1977). Diferentemente do que se pensava, mostram-se sensíveis a interrupções na reciprocidade social desde esta idade (MOORE; COHN; CAMPBELL, 2001).

Uma sensibilidade para demonstrações de afeto e trocas promovidas pelos cuidadores pode ser observada desde os três meses e meio pelo menos, com evidências de significativas relações entre os padrões de envolvimento pai/mãe-bebê e a sensibilidade do bebê para expressões emocionais (MONTAGNE; WALKER-ANDREWS, 2002). Aos três meses, mostram preferência por sorrisos com intensidade cada vez maior. Notese que os que apresentaram maior sensibilidade ao sorriso, em estudo de Kuchuck, Vibbert e Bornstein (1986), eram os que tinham mães que com maior freqüência tentavam chamar sua atenção para elas quando estavam sorrindo.

Ainda nessa idade, além da capacidade de apresentarem respostas afetivas diferenciadas para expressões faciais de alegria e tristeza, já parecem exercer certa regulação de suas emoções. O sorriso e a atenção dos bebês são regulados tanto pelo sorriso do adulto quanto pela contingência de suas respostas (D'ENTREMONT; MUIR, 1997). Esse processo de auto-regulação depende, em boa medida, da habilidade em reconhecerem expressões emocionais, que, por sua vez, é bastante facilitada pela familiaridade com a pessoa com quem interagem (KAHANAKALMAN; WALKER-ANDREWS, 2001). 
Quanto à capacidade dos bebês de produzirem expressões faciais, aos dois meses e meio, segundo Izard e cols. (1995), verifica-se uma estabilidade morfológica de expressões emocionais específicas e de respostas diferenciadas a expressões maternas de interesse, alegria, raiva, tristeza, descaso e face imóvel. Ao estudarem bebês entre um e nove meses, Izard, Huebner, Risser, McGinnes e Dougherty (1980) atestam a capacidade de eles produzirem expressões de interesse, alegria, surpresa, tristeza, raiva, repulsa, desdém e medo. Nesse sentido, as expressões faciais parecem cumprir papel fundamental como unidades expressivas, organizadas de modo flexível em configurações que transmitem mensagens sobre o estado interior e as intenções de bebês (WEI NBERG; TRONICK, 1994).

Sua exibição de sorrisos parece sofrer influência de fatores inerentes ao contexto em que se encontram e às capacidades perceptivas que detém. Diferentes tipos de sorriso podem ser exibidos por bebês de um a seis meses, durante diferentes momentos da interação com a mãe (MESSINGER; cols., 2001). No geral, parece prevalecer um sorriso caracterizado por boca fechada, sem elevação de bochechas. Nos instantes em que a mãe está sorrindo e o bebê olhando para sua face, há predomínio do sorriso de boca aberta e elevação de bochechas, associado, de acordo com Fogel, Nelson-Goens, Hsu e Shapiro (2000), a momentos de clímax em interações positivas, em que se compartilha prazer e alegria. Segundo Messinger e cols. (2001), este sorriso apresenta tendência a aumentar com a idade, quando a mãe está sorrindo e o bebê olhando para sua face, e a declinar, na situação totalmente oposta.

Aos poucos, entre dois e seis meses, os bebês desenvolvem expectativas sociais em contextos diádicos (em nossa cultura, especialmente em trocas face-a-face, Keller, 2007). A partir, em média, dos quatro meses de vida a atividade interativa mãe-bebê começa a se dar de forma diversa incluindo outros recursos. Com seis meses, já têm uma perspectiva da história das interações anteriores. O exame das reações afetivas dos bebês entre três e seis meses, ao afeto positivo de um parceiro, durante as interações em que se engajam, indica competência social e perceptiva. Os bebês discriminam objetos e pessoas e sorriem mais para estas do que para aqueles (ELLSWORTH; MUIR; HAINS, 1993). Reforçando a importância das trocas afetivas, o afeto positivo dos pais aos três meses de idade predisse o afeto positivo dos bebês aos seis meses, no estudo de Forbes, Cohn, Allen e Lewinsohn, (2004).

Aos quatro meses, os bebês olham mais para expressões faciais de alegria do que raiva (LABARBERA; IZARD; VIETZE et al., 1976), e aos cinco, sorriem mais para as expressões de alegria do que de tristeza, independentemente de virem acompanhadas ou não de expressão vocal correspondente. No entanto, se as faces são encobertas, não ocorrem respostas diferenciadas para vozes (D'ENTREMONT; MUIR, 1999). Aos 
sete meses, mas não aos cinco, são considerados como aptos a detectar correspondências entre expressões faciais e vocais com base no significado afetivo (SOKEN; PICK, 1992/1999; WALKER-ANDREWS, 1986). Se desde os primeiros meses de vida exibem sorrisos diferenciados, só a partir dos seis meses há evidências de que, ao sorrir, os bebês experimentam tipos qualitativamente diferentes de prazer. Interpreta-se que a mesma movimentação facial, o sorriso, pode refletir diferentes emoções positivas dependendo da pessoa com quem interagem e da dinâmica do processo social. Assim, a experiência emocional de bebês dessa idade é considerada mais complexa do que se supunha há alguns anos atrás (FOGEL; cols., 2000).

Indubitavelmente, é atribuída grande importância às trocas afetivas em etapas iniciais, tanto para a formação de apego em bebês, quanto para o seu desenvolvimento emocional (BOWLBY, 2002). Entende-se que alterações no ambiente familiar, como casos de depressão de um dos pais, podem aumentar o risco de psicopatologias em crianças (CUMMINGS, 1995). Da mesma forma, um quadro desfavorável como este favorece o aparecimento de afeto negativo em maior proporção e os bebês mostram-se menos positivos durante as interações de que participam (CAMPBELL; cols., 1995; COHN; cols., 1990; FORBES ; cols., 2004). O papel dessas interações, envolvendo expressões faciais de emoção, desde o nascimento, é considerado como decisivo para o processo de desenvolvimento da referência social que se apresenta em torno dos 10 meses (MCARTHUR; BARON, 1983; WALKER-ANDREWS, 1997). Entre os oito e 10 meses, Venezia, Messinger, Thorp e Mundy (2004) registram o surgimento do sorriso que designam como antecipatório. Foi observado um aumento da probabilidade dos bebês nessa idade, olharem para o observador, enquanto sorriam para um objeto. Os autores especulam tratar-se do desenvolvimento de uma habilidade para comunicar afeto positivo relacionado a algo que não à pessoa com quem estão interagindo.

Essa é uma fase de outra transição importante com transformação e ampliação das formas de trocas com os outros. Na medida em que a intersubjetividade secundária se estabelece, se mostra cada vez mais nítida a cooperação entre os parceiros. Por volta dos nove meses, o bebê consegue se tornar consciente de que compartilha seu mundo com as outras pessoas e é possível observar uma fluência completa na interação pessoa-pessoa-objeto. O bebê começa a assumir, para Rochat e Striano (1999), uma perspectiva intencional em relação às pessoas. Desde os três meses os bebês são capazes de exploração sistemática e deteç̧ão de informação de movimento que especifica intencionalidade em entidades animadas que se movem independentemente do eu. No entanto, é só a partir dos sete meses que mostram sinais de uma perspectiva intencional 
que ultrapassa uma mera discriminação perceptual de dinâmica de movimentos.

Próximos de completarem um ano, as brincadeiras envolvendo pais-bebê são mais variadas e duradouras e os sorrisos dos bebês configuram certa tipologia. Assim, como observado por Dickson e cols. (1997), os sorrisos além de variarem com o contexto, mudam de configuração a depender da forma e tipo de brincadeira e do parceiro com quem estão brincando.

Em síntese, a partir de manifestações iniciais de sorriso, em bebês recémnascidos, observa-se um desenvolvimento nessa manifestação emocional ao longo do primeiro ano. De fato, os comportamentos e as expressões faciais presentes durante as trocas afetivas passam por mudanças, envolvendo maior diferenciação, reciprocidade e a aprendizagem de condições que permitem a antecipação e o compartilhamento.

\section{Características dos estudos selecionados e lacunas observadas}

Algumas características dos estudos sobre expressões faciais de emoção selecionados nesse trabalho merecem ser examinadas mais diretamente, já que sinalizam tendências e carências que podem nortear a formulação de futuras pesquisas. Nesse sentido, são apreciadas a abrangência da observação em termos de tempo (se estudo transversal ou longitudinal), a faixa etária dos bebês participantes e o ambiente em que foi feita a observação.

Em primeiro lugar, como pode ser visto no quadro 1, a maioria desses estudos (cerca de $71 \%$ ) é transversal, focalizando, portanto, momentos específicos do desenvolvimento. Em geral, tratam de faixas etárias acima dos três meses (62\%, aproximadamente), e, por fim, são realizados em laboratório (cerca de 68\%). 


\begin{tabular}{|c|c|c|c|c|}
\hline No. & Autor(es)-Ano & Trans/Long & $\begin{array}{c}\text { Faixa etária } \\
\text { (meses) }\end{array}$ & Local \\
\hline 1 & Bigelow et al. (1996) & $\mathrm{T}$ & 4,6 e 8 & laboratório \\
\hline 2 & Bigelow (1998) & $\mathrm{T}$ & 4 & laboratório \\
\hline 3 & Campbell et al. (1995) & $\mathrm{L}$ & 2,4 e 6 & casa \\
\hline 4 & Cohn et al. (1990) & $\mathrm{T}$ & 2 & casa \\
\hline 5 & Cohn \& Tronick (1987) & $\mathrm{T}$ & 3,6 e 9 & laboratório \\
\hline 6 & D'Entremont \& Muir (1997) & $\mathrm{T}$ & 3 & laboratório \\
\hline 7 & D'Entremont \& Muir (1999) & $\mathrm{T}$ & 5 & laboratório \\
\hline 8 & Dickson et al. (1997) & $\mathrm{T}$ & 12 & casa \\
\hline 9 & Ellsworth, Muir \& Hains (1993) & $\mathrm{T}$ & 3 e 6 & laboratório \\
\hline 10 & Fogel et al. (2000) & $\mathrm{T}$ & 6 e 12 & laboratório \\
\hline 11 & Forbes et al. (2004) & $\mathrm{L}$ & 3 e 6 & laboratório \\
\hline 12 & Haviland \& Lelwica (1987) & $\mathrm{T}$ & (10 semanas) & laboratório \\
\hline 13 & Izard et al. (1980) & $\mathrm{T}$ & 1 a 9 & clínica \\
\hline 14 & Izard et al. (1995) & $\mathrm{L}$ & 2,5 a 9 & laboratório \\
\hline 15 & Kahana-Kalman \& Walker-Andrews (2001) & $\mathrm{T}$ & 3,5 & laboratório \\
\hline 16 & Kawakami et al. (2007) & $\mathrm{L}$ & $0 \mathrm{a} 6$ & casa \\
\hline 17 & Kaye \& Fogel (1980) & $\mathrm{L}$ & $\begin{array}{c}6,13 \text { e } 26 \\
\text { (semanas) }\end{array}$ & casa \\
\hline 18 & Kuchuck, Vibbert \& Bornstein (1986) & $\mathrm{T}$ & 3 & casa/lab \\
\hline 19 & LaBarbera et al. (1976) & $\mathrm{T}$ & 4,6 & laboratório \\
\hline 20 & Lavelli \& Fogel (2005) & $\mathrm{L}$ & 0 a 3 & casa \\
\hline 21 & Landau (1977) & $\mathrm{T}$ & $2,4,7$ e 11 & casa \\
\hline 22 & Legerstee \& Varghese (2001) & $\mathrm{T}$ & 2 e 3 & laboratório \\
\hline 23 & Messinger et al. (2001) & $\mathrm{L}$ & $1 \mathrm{a} 6$ & laboratório \\
\hline 24 & Messinger et al. (2002) & $\mathrm{T}$ & $(5 \mathrm{a} 106 \mathrm{~h})$ & maternidade \\
\hline 25 & Montagne \& Walker-Andrews (2002) & $\mathrm{T}$ & 3,5 & laboratório \\
\hline 26 & Moore, Cohn \& Campbell (2001) & $\mathrm{L}$ & 2,4 e 6 & casa \\
\hline 27 & Nelson \& Horowitz (1983) & $\mathrm{T}$ & 5 & laboratório \\
\hline 28 & Soken \& Pick (1992) & $\mathrm{T}$ & 7 & laboratório \\
\hline 29 & Soken \& Pick (1999) & $\mathrm{T}$ & 7 & laboratório \\
\hline 30 & Symons \& Moran (1994) & $\mathrm{L}$ & $2,3,4$ e 5 & laboratório \\
\hline 31 & van Beek, Hopkins \& Joekoma (1994) & $\mathrm{T}$ & entre 6 e 18 & laboratório \\
\hline 32 & Venezia et al. (2004) & $\mathrm{L}$ & 8,10 e 12 & laboratório \\
\hline 33 & Walker-Andrews (1986) & $\mathrm{T}$ & 5,7 & laboratório \\
\hline 34 & Weinberg \& Tronick (1994) & $\mathrm{T}$ & 6 & laboratório \\
\hline
\end{tabular}

Uma apreciação da situação encontrada indica que a produção de resultados em estudos empíricos, apesar de vasta, apresenta lacunas. A necessidade de mais estudos longitudinais que auxiliam uma compreensão em termos da ontogênese é clara. Vários dos estudos citados, embora permitam a inferência de uma seqüência evolutiva, não a investigaram efetivamente, por serem transversais. A importância da experimentação em laboratório é inequívoca, mas é necessário voltar ao ambiente natural para investigar a microgênese e a ontogênese de processos sobre os quais 
foram formuladas e testadas hipóteses. Como apontam Chen e Miller (2005),

Muitas questões evolutivas fundamentais, como se o fenômeno apresenta continuidade de desenvolvimento ou mudança e como influências pessoais e contextuais iniciais contribuem para o desenvolvimento social e cognitivo posteriormente, só podem ser endereçadas pela pesquisa longitudinal ( $p .1)$.

Finalmente, a análise dos estudos levantados revela que as etapas iniciais da vida, anteriores ao terceiro mês de idade, pela sua importância no desenvolvimento de capacidades cognitivas, sociais e emocionais, carecem de maior atenção.

\section{Algumas considerações finais e possíveis desdobramentos}

O estudo das expressões emocionais em bebês revela-se crucial e instigante. Acredita-se que desde muito cedo manifestam suas emoções, fundamentalmente na face, já que outros possíveis canais, como o da comunicação verbal, ainda não estão em funcionamento. Por outro lado, não se valem, como os adultos, de mecanismos de dissimulação que mascaram as emoções. Assim, pode-se considerar, como Messinger (2002), que uma alternativa consistente para se conhecer os processos emocionais e seu desenvolvimento é a investigação de expressões faciais nas etapas iniciais da vida.

Para além das diferentes expressões, evidências de variações de uma mesma expressão, como nos sorrisos de diferentes tipos, parecem associadas a significados diferenciados. Tais variações são registradas através da observação de situações em que os bebês produzem expressões faciais distintas e dos escores atribuídos por juízes (observadores ingênuos) à intensidade dessas expressões. O fato é que, segundo autores como Messinger (2002), Izard (1990) e Ekman (1993), e a par de todo o esforço realizado, sabe-se pouco acerca da relação entre as expressões faciais de bebês e sua experiência emocional, o que aponta para a necessidade de novos estudos.

Alguns aspectos do problema são alvos de interpretações convergentes, como o entendimento de que a manifestação de afeto positivo à sua volta, e as trocas afetivas com a mãe se refletem nas expressões emocionais do bebê. $\mathrm{Na}$ medida em que, ele começa a perceber e antecipar os comportamentos emocionais do outro e atribuir-lhes significados, as interações afetivas são vistas como contexto privilegiado de desenvolvimento cognitivo e socioemocional. Pode-se pensar, portanto, que ainda que as expressões faciais de certas emoções consideradas básicas como a alegria e a raiva (EKMAN, 2003) possam estar prontas ao nascer, tem-se que, de certo modo, aprender a manifestá-las. 
A questão da origem das expressões faciais de emoção, contudo, continua motivando grande discussão entre teóricos e pesquisadores. Expressa, muitas vezes, como uma dicotomia inato $x$ aprendido ou biologia $x$ cultura, permanece carente de consenso, não contribuindo verdadeiramente para 0 avanço do conhecimento. Perspectivas interacionistas que procuram conciliar os pressupostos de uma base biológica de predisposições inatas com um desenvolvimento que se orienta por essas predisposições e envolve aquisição de capacidades construídas pelo sujeito, mostram-se oportunas.

Nesse sentido, pode-se pensar em uma necessária articulação de aspectos genéticos, biológicos, socioculturais, históricos e filogenéticos contemplados em uma abordagem sociocultural e evolucionista do desenvolvimento humano, como propõe Seidl-de-Moura (2005). Um dos pontos centrais dessa proposta se refere aos diferentes planos de gênese (a evolução da espécie, a história cultural e a ontogênese) a serem analisados para se estudar um fenômeno humano complexo. O homem é visto como um ser racional, produto da filogênese e da história cultural, que ao se desenvolver constitui e é constituído pela cultura. Sendo assim, revela-se, como definem Bussab e Ribeiro (1998), como um serbiologicamente cultural.

Observando a dispersão e pouca explicitação teórica dos estudos revistos, o que se propõe, em contrapartida, é a adoção de uma perspectiva que procure integrar os condicionantes biológicos e socioculturais, contemplando a ontogênese sem desconsiderar a história filogenética da espécie. Esse é o desafio da psicologia do desenvolvimento contemporânea. Iniciativas nesse sentido estão sendo feitas por autores evolucionistas (BJ ORKLUND; PELLEGRINI, 2002) e parecem promissoras. Como sugere Izard (1993), a ativação de um sistema de emoção depende de quatro outros sistemas: neural, sensório-motor, motivacional e cognitivo. O desenvolvimento desses quatro sistemas permitiu, ao longo da evolução, a ativação de sistemas de emoção envolvidos na adaptação e na sobrevivência. O sistema neural estabelece a base para a experiência emocional; o sensório-motor age nas interações criança-adulto, proporcionando a comunicação e o estabelecimento de vínculos; o sistema motivacional ativa emoções em condições específicas; e o cognitivo ativa emoções quando acontecimentos externos ou internos exigem comparação, categorização, julgamento. Desse modo, pode-se afirmar que na perspectiva evolucionista o desenvolvimento emocional é considerado integrado a outras esferas do desenvolvimento e parte de um processo que foi produto da seleção natural.

As apreciações apresentadas, pautadas em reflexões baseadas na literatura disponível, justificam e ressaltam a importância de mais estudos nesse campo. Muitas são as questões que permanecem sem respostas ou com respostas parciais. Aos pesquisadores interessados cabe identificá-las 
para que suas iniciativas se somem ao conhecimento acumulado e promovam um melhor entendimento do desenvolvimento emocional dos seres humanos. Para abordá-las, perspectivas metodológicas longitudinais e em ambiente natural parecem necessárias para articularem-se às iniciativas de estudos transversais até agora predominantes. A disponibilidade de novas e sofisticadas técnicas de análise estatística de dados longitudinais (BLOZIS; CONGER; HARRING, 2007; CARD; LITTLE, 2007, CHEN; MILLER, 2005; MCCARTNEY, BURCHINAL; BUB, 2006) abre novas e fascinantes perspectivas nessa área.

\section{Referências Bibliográficas}

ALONSO, A. L. et al. Neuropsicología de la percepción y la expresión facial de emociones: estudios com niños y primates no humanos. Anales de Psicologia, Murcia, v. 20, n. 2, p. 241-259, 2004.

BIGELOW, A. E. Infant's sensitivity to familiar imperfect contingencies in social interaction. I nfant Behavior and Development, Nova York, v. 21, n. 1, p. 149-162, 1998.

BIGELOW, A. E.; MACLEAN, B. K.; MACDONALD, D. Infant's response to live and replay interactions with self and mother. Merril-Palmer Quarterly, Detroit, v. 42, n. 4, p. 596-611, 1996.

BJORKLUND, D. F.; PELLEGRINI, A. D. The origins of human nature: evolutionary developmental psychology. Washington, D.C., American Psychological Association, 2002.

BLOZIS, S. A.; CONGER, K. J. ; HARRING, J. R. Nonlinear latent curve models for multivariate longitudinal data. International Journal of Behavioral Development, Melbourne, v. 31, n. 4, p. 340-346, 2007.

BOWLBY, J. Apego: A natureza do vínculo. São Paulo: Martins Fontes, 2002.

BRAUNGART-RIEKER, J.; GARWOOD, M. M., POWERS; B. P.; NOTARO, P. C. Infant affect and affect regulation during the still-face paradigm with mothers and fathers: The role of infants characteristics and parental sensitivity. Developmental Psychology, Washington, v. 34, p. 14281437, 1998.

BRAUNGART-RIEKER, J. M.; GARWOOD, M. M.; POWERS, B. P.; WANG, X. Parental sensitivity, infant affect, and affect regulation: predictors of later attachment. Child Development, Oxford, v. 72, n. 1, p. 252-270, 2001. BRONFENBRENNER, U. L. A ecologia do desenvolvimento humano: experimentos naturais e planejados. Porto Alegre: Artes Médicas, 1996. BUSSAB, V. S. R.; RIBEIRO, F. L. Biologicamente cultural. In: L. SOUZA; M. F. Q. FREITAS; M. M. P. RODRIGUES (Orgs.). Psicologia: reflexões (im) pertinentes. São Paulo: Casa do Psicólogo, 1998, p. 175-224. 
CARD, N. A.; LITTLE, T. D. Longitudinal modeling of developmental processes. International Journal of Behavioral Development, Melbourne, v. 31, n. 4, p. 297-302, 2007.

CAMPBELL, S. B.; COHN, J. F.; MEYERS, T. Depression in first-time mothers: mother-infant interaction and depression chronicity.Developmental Psychology, Washington, v. 31, n. 3, p. 349357, 1995.

CHEN, X.; MILLER, J. G. Introduction to longitudinal research on human development: approaches, issues and new directions. International Society for the Study of Behavioural Development Newsletter, Melbourne, v. 2, n. 48, p. 1-1, 2005.

COHN, J. F.; CAMPBELL, S. B.; MATIAS, R.; HOPKINS, J. Face-to-face interactions of postpartum depressed and non-depressed mother-infant pairs at 2 months. Developmental Psychology, Washington, v. 26, n. 1, p. 15-23, 1990.

COHN, J. F.; TRONICK, E. Z. Mother-infant face-to-face interaction: the sequence of dyadic states at 3,6 , e 9 months. Developmental Psychology, Washington, v. 23, n. 1, p. 68-77, 1987.

CUMMINGS, E. M. Security, emotionality, and parental depression: a commentary. Developmental Psychology, Washington, v. 31, n. 3, p. 425-427, 1995.

D'ENTREMONT, B.; MUIR, D. W. Five-month-olds' attention and affective responses to still-faced emotional expressions. Infant Behavior and Development, Nova York, v. 20, n. 4, p. 563-568, 1997.

D'ENTREMONT, B.; MUIR, D. W Infant's responses to adult happy and sad vocal and facial expressions during face-to-face interactions. Infant Behavior and Development, Nova York, v. 22, n. 4, p. 527-539, 1999.

DICKSON, K. L.; WALKER, H.; FOGEL, A. The relationship between smile type and play type during parent-infant play. Developmental Psychology, Washington, v. 33, n. 6, p. 925-933, 1997.

EKMAN, P. Facial Expression and Emotion. American Psychologist, Washington, v. 48, p. 384-392, 1993. Disponível em: http://www.paulekman.com Acesso em: outubro de 2005.

EKMAN, P. Emotions revealed: recognizing faces and feelings to improve communication and emotional life. NY: Times Books, 2003. ELLSWORTH, C. P.; MUIR, D. W.; HAINS, S. M. J. Social competence and person-object differentiation: an analysis of the still-face effect.Developmental Psychology, Washington, v. 29, n. 1, p. 63-73, 1993.

FOGEL, A.; NELSON-GOENS, C.; HSU, H.; SHAPIRO, A. F. Do different infant smiles reflect different positive emotions? Social Development, Oxford, v. 9, n. 4, p. 497-520, 2000.

FORBES, E. E.; COHN, J. F.; ALLEN, N. B.; LEWINSOHN, P. M. Infant affect during parent-infant interaction at 3 and 6 months: differences 
between mothers and fathers and influence of parent history of depression. Infancy, Cambridge, v. 5, n. 1, 61-84, 2004.

HARKNESS, S. C.; SUPER, C. M. Parental ethnotheories in action. In: I. E. SIGEL; A. V. MCGILLICUDDY-DELISI; J. J. GOODNOW (Orgs.), Parental belief systems: the psychological consequences for children. Hillsdale, NJ e Hove, UK: Lawrence Erlbaum Associates, 1992, p. 373-392.

HAVILAND, J. M.; LELWICA, M. The induced affect response: 10-week-old infants' responses to three emotion expressions. Developmental Psychology, Washington, v. 23, n. 1, p. 97-104, 1987.

IZARD, C. E. Facial expressions and the regulation of emotions. J ournal of Personality and Social Psychology, Washington, v. 58, n. 3, p. 487498, 1990.

IZARD, C. E. Four systems for emotion activation: cognitive and noncognitive processes. Psychological Review, Washington, v. 100, p. 68-90, 1993.

IZARD, C. E.; FANTAUZZO, C. A.; CASTLE, J. M.; HAYNES, O. M.; RAYIAS, M. F.; PUTNAM, P. H. The ontogeny and significance of infants' facial expressions in the first 9 months of life. Developmental Psychology, Washington, v. 31, n. 6, p. 997-1013, 1995.

IZARD, C. E.; HUEBNER, R. R.; RISSER, D.; MCGINNES, G.; DOUGHERTY, $L$. The young infant's ability to reproduce discrete emotion expressions.Developmental Psychology, Washington, v. 16, p. 132140, 1980.

KAHANA-KALMAN, R.; WALKER-ANDREWS, A. The role of person familiarity in young infants'perception of emotional expressions. Child Development, Oxford,v. 72, n. 2, p. 352-369, 2001.

KAWAKAMI, K.; TAKAI-KAWAKAMI, K.; TOMONAGA, M.; SUZUKI, J.; KUSAKA, F.; OKAI, T. Spontaneous smile and spontaneous laugh: An intensive longitudinal case study. I nfant Behavior ; Development, Nova York, v. 30, p. 146-152, 2007.

KAYE, K.; FOGEL, A. The temporal structure of face-to-face communication between mothers and infants. Developmental Psychology, Washington, v. 16, p. 454-464, 1980.

Keller, H. , 2007.

KUCHUCK, A.; VIBBERT, M.; BORNSTEIN, M. H. The perception of smiling and its experiential correlates in three-month-old infants. Child Development, Oxford, v. 57, n. 4, p. 1054-1061, 1986.

LABARBERA, J. D.; IZARD, C. E.; VIETZE, P.; PARISI, S. A. Four- and sixmonth-old infants' visual responses to joy, anger, and neutral expressions. Child Development, Oxford, v. 47, p. 535-538, 1976.

LEGERSTEE, M.; VARGHESE, J. The role of maternal affect mirroring on social expectancies in three-month-old infants. Child Development, Oxford, v. 72, n. 5, 1301-1313, 2001. 
LANDAU, R. Spontaneous and elicited smiles and vocalizations of infants in four Israeli environments. Developmental Psychology, Washington, v. 13, n. 4, p. 389-400, 1977.

LAVELLI, M.; FOGEL, A. Developmental changes in the relationship between the infant's attention and emotion during early face-to-face communication: the 2-month transition. Developmental Psychology, Washington, v. 41, n. 1, p. 265-280, 2005.

MCARTHUR, L. Z.; BARON, R. M. Toward an ecological theory of social perception. Psychological Review, Washington, v. 90, p. 215-238, 1983.

MCCARTNEY, K.; BURCHINAL, M. R.; BUB, K. L. Best practices in quantitative methods for developmentalists. Monographs of the Society for Research in Child Development, Oxford, v. 71, n. 3, serial no. 285, 2006.

MESSINGER, D. S. Positive and negative: Infant facial expressions and emotions. Current Directions in Psychological Science, Washington, v. 11, n. 1, p. 1-6, 2002.

Disponível em: http://www.psy.miami.edu/faculty/dmessinger . Acesso em: setembro de 2005.

MESSINGER, D.; DONDI, M.; NELSON-GOENS, G. C.; BEGHI, A.; FOGEL, A.; SIMION, F. How sleeping neonates smile. Developmental Science, v. $5, \quad$ n. $1,48-54,2002$. Obtido em http://www. blackwellsynergy.com/ em agosto de 2005.

MESSINGER, D. S., FOGEL, A. ; DICKSON, K. L. All smiles are positive, but some smiles are more positive than others. Developmental Psychology, Washington, v. 37, n. 5, p. 642-653, 2001.

MONTAGNE, D. P. F.; WALKER-ANDREWS, A. Mothers, fathers, and infants: the role of person familiarity and parental involvement in infants perception of emotion expressions. Child Development, Oxford, v. 73, n. 5, p. 1339-1352, 2002.

MOORE, G. A.; COHN, J. F.; CAMPBELL, S. B. Infant affective responses to mother's still face at 6 months differentially predict externalizing and internalizing behaviors at 18 months. Developmental Psychology, Washington, v. 37, n. 5, p. 706-714, 2001.

NELSON, C. A.; HOROWITZ, F. D. The perception of facial expressions and stimulus motion by 2 - and 5-month-old infants using holographic stimuli. Child Development, Oxford, v. 54, p. 868-877, 1983.

ROCHAT, P. The infant's world. Cambridge: Harvard University Press, 2001.

ROCHAT, P.; STRIANO, T. Social-cognitive development in the first year. In: P. ROCHAT (Org.), Early social cognition understanding others in the first months of life. Mahwah, New Jersey ; London: Lawrence Erlbaum, 1999, p. 3-34. 
SEIDL-DE-MOURA, M. L.; RIBAS, A. P.; SEABRA, K. C.; PESSÔA, L. F.; NOGUEIRA, S. E.; MENDES, D. M. L. F.; ROCHA, S. B.; VICENTE, C. C. Interações mãe-bebê de um e cinco meses de díades urbanas: aspectos afetivos, comportamentos, complexidade e sistemas parentais predominantes. Psicologia. Reflexão e Crítica, Porto Alegre. No prelo.

SEIDL-DE-MOURA, M. L. Bases para uma psicologia do desenvolvimento sociocultural e evolucionista. In: F. A. R. Pontes, C. M. C. Magalhães, R. C. S. Brito e W. L. B. Martin (Orgs.). Temas pertinentes à construção da Psicologia contemporânea. Belém: EDUFPA, 2005, p. 15-41.

SOKEN, N. H.; PICK, A. D. Intermodal perception of happy and angry expressive behaviors by seven-month-old infants. Child Development, Oxford, v. 63, p. 787-795, 1992.

SOKEN, N. H.; PICK, A. D. Infants' perception of dynamic affective expressions: do infants distinguish specific expressions? Child Development, Oxford, v. 70, n. 6, p. 1275-1282, 1999.

STACK, D. M.; MUIR, D. W. Tactile stimulation as a component of social interchange: New interpretations for the still-face effect. British J ournal of Developmental Psychology, Londres, v. 8, p. 131-145, 1990.

SYMONS, D. ; MORAN, G. Responsiveness and dependency are different aspects of social contingencies: An example from mother and infant smiles. Infant Behavior and Development, Nova York, v. 17, p. 209214, 1994.

TRONICK, E. Z. Emotions and emotional communication in infants. American Psychologist, Washington, v. 44, p. 112-119, 1989.

VAN BEEK, Y.; HOPKINS, B.; JOEKOMA, J. B. Development of communicative behaviors in preterm infants: the effects of birth weight status and gestational age. Infant Behavior and Development, Nova York, v. 17, p. 107-118, 1994.

VENEZIA, M.; MESSINGER, D. S.; THORP, D.; MUNDY, P. Timing changes: the development of anticipatory smiling. I nfancy, Cambridge, v. 6, n. 3, p. 397-406, 2004.

WALKER-ANDREWS, A. S. Intermodal perception of expressive behaviors: relation of eye and voice? Developmental Psychology, Washington, $v$. 22, n. 3, p. 373-377, 1986.

WALKER-ANDREWS, A. S. Infant's perception of expressive behaviors: Differentiation of multimodal information. Psychological Bulletin, Washington, v. 121, p. 437-456, 1997.

WEINBERG, M. K.; TRONICK, E. Z. Beyond the face: An empirical study of infant affective configurations of facial, vocal, gestural, and regulatory behaviors. Child Development, Oxford, v. 65, p. 1503-1515, 1994.

\section{Endereço para correspondência}

Deise Maria Leal Fernandes Mendes

Universidade do Estado do Rio de Janeiro-UERJ, Rua São Francisco Xavier, 524, Bloco F, 
10 andar, Instituto de Psicologia, Programa de Pós-Graduação em Psicologia Social, CEP: 20550-013, Rio de Janeiro, RJ, Brasil.

Endereço eletrônico: deisefmendes@gmail.com

Maria Lucia Seidl de Moura

Universidade do Estado do Rio de Janeiro-UERJ, Rua São Francisco Xavier, 524, Bloco F, 10ㅇaㅁ andar, Instituto de Psicologia, Programa de Pós-Graduação em Psicologia Social,

CEP: 20550-013, Rio de Janeiro, RJ, Brasil.

Endereço eletrônico: mlseidl@gmail.com

Recebido em: 28/03/2008

Aceito para publicação em: 12/11/2008

Editor responsável: Ariane Patrícia Ewald

\section{Notas}

* Psicóloga formada pela UFRJ, Mestre em Psicologia Social pelo Programa de PósGraduação em Psicologia Social da UERJ, e doutoranda do mesmo Programa. É membro do grupo de pesquisas Interação Social e Desenvolvimento do Diretório de Grupos de Pesquisas do CNPq.

** Psicóloga, doutora em Psicologia Cognitiva pela Fundação Getúlio Vargas, pósdoutorado na USP, Livre-docente pela UFRJ, Pesquisadora do CNPq, é professora titular da UERJ, atuando na graduação e no Programa de Pós-Graduação em Psicologia Social do Instituto de Psicologia. 